\title{
No association between exposure to perfluorinated compounds and congenital cryptorchidism: a nested case-control study among 215 boys from Denmark and Finland
}

\author{
Dorte Vesterholm Jensen ${ }^{1}$, Jeppe Christensen ${ }^{2}$, Helena E Virtanen ${ }^{3}$, Niels E Skakkebæk ${ }^{2}$, \\ Katharina M Main ${ }^{2}$, Jorma Toppari ${ }^{3}$, Christine W Veje ${ }^{2}$, Anna-Maria Andersson², \\ Flemming Nielsen ${ }^{1}$, Philippe Grandjean ${ }^{1}$ and Tina Kold Jensen ${ }^{1,2}$ \\ ${ }^{1}$ Department of Environmental Medicine, University of Southern Denmark, J.B. Winsløws Vej 17, 5000 Odense, \\ Denmark, ${ }^{2}$ University Department of Growth and Reproduction, Rigshospitalet, Copenhagen, Denmark and \\ ${ }^{3}$ Departments of Physiology and Paediatrics, University of Turku, Turku, Finland
}

Correspondence should be addressed to T K Jensen at Department of Environmental Medicine, University of Southern Denmark; Email: tkjensen@health.sdu.dk

\begin{abstract}
Geographical differences in the occurrence of diseases in male reproductive organs, including malformation in reproductive tract, between Denmark and Finland have been reported. The reason for these differences is unknown, but differences in exposure to chemicals with endocrine-disrupting abilities have been suggested. Among these chemicals are perfluoro-alkylated substances (PFASs), a group of water- and grease-repellent chemicals used in outdoor clothes, cookware, food packaging, and textiles. In this study, we, therefore, investigated differences in PFAS exposure levels between Denmark and Finland and the association between cord blood PFAS levels and congenital cryptorchidism. Boys from a joint ongoing prospective birth cohort study were included. We analyzed PFAS levels in cord blood serum samples collected from 29 Danish boys with congenital cryptorchidism, 30 healthy Danish matched controls recruited from 1997 to 2001, 30 Finnish cases, and 78 Finnish healthy matched controls recruited from 1997 to 1999. Additionally, 48 Finnish cases recruited from 2000 to 2002 were included. Perfluorooctanoic acid (PFOA) and perfluorooctanesulfonic acid (PFOS) were detected in all the 215 Danish and Finnish cord blood samples with significantly higher levels being observed in the Danish samples (medians: PFOA, $2.6 \mathrm{ng} / \mathrm{ml}$ and PFOS, $9.1 \mathrm{ng} / \mathrm{ml}$ ) than in the Finnish samples (medians: PFOA, $2.1 \mathrm{ng} / \mathrm{ml}$ and PFOS, $5.2 \mathrm{ng} / \mathrm{ml}$ ). We found no associations between cord blood PFOA and PFOS levels and congenital cryptorchidism after adjustment for confounders. Our data indicate that women in Denmark and Finland are generally exposed to PFOA and PFOS but there are differences in exposure levels between countries. We found no statistically significant association between cord blood PFOA and PFOS levels and congenital cryptorchidism; however, our study was small and larger studies are warranted.

Reproduction (2014) 147 411-417
\end{abstract}

This paper forms part of a special issue of Reproduction on Endocrine Disrupters. This article was presented at the 7 th Copenhagen Workshop on Endocrine Disrupters, 28-31 May 2013. The meeting was supported by the Danish Ministry of the Environment - Environmental Protection Agency as an activity under the Danish Centre on Endocrine Disrupters. Publication of this special issue has been supported by the Society for Reproduction and Fertility. The opinions or views expressed in this special issue are those of the authors, and do not necessarily reflect the opinions or recommendations of the Danish Ministry of the Environment - Environmental Protection Agency or the Society for Reproduction and Fertility. The Guest Editors for this special issue were Anna-Maria Andersson, Hanne Frederiksen, Niels Erik Skakkebæk, Rigshospitalet, Denmark, Kenneth M Grigor, Western General Hospital, Edinburgh, UK and Jorma Toppari, University of Turku, Finland.

\section{Introduction}

Perfluoro-alkylated substances (PFASs) are a group of water- and grease-repellent chemicals, which makes them suitable for use in a wide range of applications, e.g. in outdoor clothes, cookware, food packaging, furniture treatment, textiles, and carpets (Jensen \& Leffers 2008). PFASs are widely used, have a long elimination half-life, and bioaccumulate. Both perfluorooctanoic acid (PFOA) and perfluorooctanesulfonic acid (PFOS) can generally be detected in human serum in Western countries (Lindstrom et al. 2011). Moreover, PFASs cross placenta and are found in newborns and cord blood (Lindstrom et al. 2011). PFOA exposure reduces testosterone levels in rats, and both PFOA and PFOS affect Leydig cell function through different modes of 
action in rats and humans (Ye et al. 2011). Information on the developmental effects of PFOS and PFOA exposure in humans has been inconsistent (Olsen et al. 2009). PFOA has been reported to be associated with low birth weight, although the results are conflicting (Fei et al. 2007, Stein et al. 2009, Washino et al. 2009). High PFOS exposure in adult men has been shown to be associated with low testosterone levels (Joensen et al. 2013). To our knowledge, no studies have investigated the association between PFAS exposure in mothers and cryptorchidism (undescended testis) in the offspring.

We conducted a nested case-control study within a Danish and a Finnish birth cohort study to investigate possible differences in exposure levels across countries and to associate PFAS levels in cord blood with congenital cryptorchidism at birth among 107 cases and 108 controls.

\section{Subjects and methods}

\section{Cohorts}

Cases and controls were sampled from an ongoing joint prospective birth cohort study conducted at the University Hospital of Copenhagen (Rigshospitalet and Hvidovre Hospital), Denmark, and Turku University Hospital, Finland. Pregnant women were recruited from 1997 to 2001 in Denmark and from 1997 to 1999 in Finland, and their children were examined at birth and 3 months of age using standardized procedures. In Finland, additional cases with cryptorchidism were recruited at birth from the total hospital cohort during 1997-2002. In the Finnish study carried out from 2000 to 2002, only boys with cryptorchidism and two controls (matched by date of birth, gestational age, parity, maternal diabetes, and smoking status) and additionally every tenth healthy boy of the prospective cohort (random healthy controls) were followed until the age of 18 months due to financial restrictions. The prospective cohort (antenatal recruitment, inclusion criteria, and clinical examinations) has been described in detail previously (Boisen et al. 2004). Study design, questionnaires, and clinical examinations were strictly standardized and controlled in repetitive workshops. We have previously reported that the prevalence of congenital cryptorchidism is higher in Denmark than in Finland, primarily due to there being more cases of transient cryptorchidism and mild forms of cryptorchidism (Boisen et al. 2004).

A total of 29 singleton Danish boys with congenital cryptorchidism and available cord blood samples were included and frequency matched by year of birth to 30 healthy singleton controls with available cord blood samples and follow-up data. A total of 30 Finnish singleton cases and 78 Finnish frequency-matched healthy singleton controls recruited from 1997 to 1999 were included. Additionally, 48 Finnish singleton cases recruited from 2000 to 2002 with available cord blood samples were included.

Testicular position was determined at birth using the examination technique and definition of cryptorchidism developed by Scorer (1964). Testicular position was divided into six categories (non-palpable, inguinal, suprascrotal, high scrotal, normal-retractile, and normal-scrotal); for this study, we used a dichotomized variable: cryptorchidism (yes or no), including high-scrotal testes in the category 'yes'. Gestational age was determined using information from routine ultrasonographies when available; otherwise, the last menstrual period was used to calculate gestational age. The variable was divided into three categories: premature $(<37$ weeks), mature (37-42 weeks), and postmature ( $>42$ weeks). Birth weight was obtained from birth records and divided into three categories for the analyses $(<2500,2500-4500$, and $>4500 \mathrm{~g})$. Information pertaining to parity was obtained through a questionnaire answered prenatally by the mother or from the hospital records (postnatally recruited cases), and it was dichotomized for this study ( 1 vs $\geq 2$ ).

\section{Analyses of PFOA and PFOS and their metabolites}

Umbilical cord blood samples were centrifuged and serum was separated and stored at $-20{ }^{\circ} \mathrm{C}$ until analysis. The PFAS analysis was carried out at Environmental Medicine, University of Southern Denmark, Denmark, using on-line solid-phase extraction followed by liquid chromatography and triple quadropole mass spectrometry (LC-MS/MS) using a previously described method (Haug et al. 2009, Vestergaard et al. 2012). The following PFASs were quantified: PFOS, PFOA, perfluorohexane sulfonic acid (PFHxS), perfluorononanoic acid (PFNA), perfluorodecanoic acid (PFDA), N-methyl-perfluorooctanoic sulfonamidoacetate (MeFOSAA), N-ethyl-perfluorooctanesulfonamidoacetate (EtFOSAA), and perfluorooctane sulfonamide (FOSA). The limit of quantification (LOQ) was $0.03 \mathrm{ng} / \mathrm{ml}$ for all the reported compounds. Values below the limit were replaced with half of the LOQ (i.e. $0.015 \mathrm{ng} / \mathrm{ml}$ ), as has been suggested previously by the guidelines of United States Environmental Protection Agency (2000).

\section{Statistical analyses}

Cord blood PFAS levels in Danish and Finnish samples collected from boys with and without cryptorchidism were compared using the Mann-Whitney $U$ non-parametric test. PFOA and PFOS levels were transformed using the natural logarithm to obtain a normal distribution. In addition, PFOA, PFOS, and PFNA levels were divided into tertiles. Differences in the distribution of potential confounders across tertiles of PFOA and PFOS exposure levels were studied. T-test or ANOVA was used to test for differences in maternal age, BMI, and birth weight, and $\chi^{2}$ test was used to test for differences in parity, country, year of birth, and smoking status between tertiles of exposure groups. As parity was a highly significant determinant of PFAS levels, analyses were also carried out for primiparas. Finally, multiple logistic regression analysis was used to calculate adjusted odds ratios (ORs) for cryptorchidism for both continuous and tertile exposure levels of PFAS controlling for birth weight, gestational age, and parity in Danish and Finnish boys separately. The tertiles of PFAS exposure levels were introduced as a categorical variable to test trends in exposure. As only a minor proportion of pregnant women had measurable levels of some PFASs in cord blood, multiple logistic regression models were conducted for only 
PFOA and PFOS exposure. Logistic regression models were checked with Pearson's goodness-of-fit test. The results are presented with 95\% Cls, and analyses were performed using STATA, version IC11 (Statacorp, TX, USA).

\section{Results}

PFOA and PFOS were detected in the cord blood of all cases and controls in both Finland and Denmark (Table 1). PFOA and PFOS levels declined with increasing year of birth in both Danish and Finnish cord blood samples and were statistically significantly higher in the Danish cord blood samples than in the Finnish cord blood samples (Table 1), if analyses were restricted to cord blood samples collected before 2000. Among the Finnish, but not the Danish boys, the levels of some PFASs differed significantly between cases and controls. However, for PFOA and PFOS, which were present in highest concentrations, no significant difference in levels between cases and controls was observed (Table 1).

Maternal parity, BMI, and smoking habits did not differ between cases and controls (data not shown). Birth weight, maternal BMI, age, and smoking status did not differ between tertiles of PFOA and PFOS exposure groups (Table 2), but more women with first pregnancies were in the third tertile of PFOA and PFOS exposure. In addition, PFOS levels were lower in the Finnish samples collected after 2000.

Table 3 summarizes crude and adjusted ORs for PFOA and PFOS exposure among boys with cryptorchidism compared with those among the controls. No significant differences were found when PFOS and PFOA exposure levels were analyzed as continuous variables. However, when PFOS and PFOA exposure levels were analyzed as tertiles, Finnish boys with cryptorchidism were significantly less likely to be in the highest tertile of PFOA exposure, as a significant trend of decreasing OR with increasing tertiles of PFOA exposure was observed
(Table 3). When the analyses were restricted to primiparous women, no differences in PFOA and PFOS exposure levels among cases and controls were found (data not shown). More Finnish cases were recruited from 2000 to 2002 during which PFOA and PFOS levels declined, and we therefore repeated the analyses among the 107 Finnish cases and controls recruited from 1997 to 1999. Adjusted OR for cryptorchidism still decreased with increasing tertiles of PFOA exposure, although no longer significant.

\section{Discussion}

We detected PFOA and PFOS in all the 215 Danish and Finnish cord blood samples with generally higher levels in the Danish samples suggesting widespread exposure to these chemicals, albeit with differences in exposure levels between countries. To our knowledge, no studies have previously measured PFAS exposure levels in the Finnish population, either in maternal blood or in cord blood. Our findings of lower levels in the Finnish than in the Danish cord blood samples are in accordance with a previous study reporting higher levels of other potential endocrine-disrupting chemicals (organochlorine pesticides, polychlorinated biphenyls, and dioxins) in breast milk of mothers in Denmark than in that of mothers in Finland (Shen et al. 2008), although levels of organotin compounds tended to be higher in Finland than in Denmark (Rantakokko et al. 2013). It has been suggested that differences in exposure to chemicals with endocrine-disrupting abilities may contribute to the geographical differences in male reproductive disorders between Denmark and Finland (Skakkebaek et al. 2001, Krysiak-Baltyn et al. 2010). Over the last 50 years, the prevalence of congenital cryptorchidism has increased in some countries, e.g. in Denmark from $<2 \%$ in 1950 s to $8.2 \%$ in 2000-2002, while the prevalence in Finland in 1997-1999 was 2.4\% (Boisen et al. 2004). Cryptorchidism, hypospadias, testis cancer, and poor

Table 1 Median (M) PFAS levels ( $\mathrm{ng} / \mathrm{ml}$ ) and 5-95 percentiles (5-95) and percent above detection levels (LOD) in all the 215 cord blood samples among cases (cryptorchidism) and controls in Denmark and Finland respectively.

\begin{tabular}{|c|c|c|c|c|c|c|c|c|c|c|c|c|c|}
\hline \multirow[b]{3}{*}{ PFAS (ng/ml) } & \multirow{3}{*}{$\begin{array}{c}\text { Percentage } \\
(>\text { LOD })\end{array}$} & \multicolumn{6}{|c|}{ Denmark } & \multicolumn{6}{|c|}{ Finland } \\
\hline & & \multicolumn{2}{|c|}{ All $(n=59)$} & \multicolumn{2}{|c|}{ Controls $(n=30)$} & \multicolumn{2}{|c|}{ Cases $(n=29)$} & \multicolumn{2}{|c|}{ All $(n=156)$} & \multicolumn{2}{|c|}{ Controls $(n=78)$} & \multicolumn{2}{|c|}{ Cases $(n=78)$} \\
\hline & & M & $5-95$ & M & $5-95$ & M & $5-95$ & M & 5-95 & M & $5-95$ & M & 5-95 \\
\hline PFOA & 100 & $2.6^{\mathrm{a}}$ & $1.4-4.4$ & 2.70 & $1.4-4.0$ & 2.4 & $1.4-4.4$ & $2.1^{\mathrm{a}}$ & $1.1-4.1$ & 2.3 & $1.2-4.8$ & 1.9 & $1.0-3.9$ \\
\hline PFOS & 100 & $9.1^{\mathrm{a}}$ & $4.8-16.4$ & 10.2 & $4.7-14.0$ & 8.9 & $5.1-17.5$ & $5.2^{\mathrm{a}}$ & $2.2-10.5$ & 5.5 & $2.5-11.1$ & 4.8 & $2.2-9.6$ \\
\hline PFHxS & 39 & 0.01 & $0.01-0.44$ & 0.02 & $0.02-0.2$ & 0.02 & $0.02-0.79$ & 0.01 & $0.01-0.3$ & $0.02^{b}$ & $0.02-0.34$ & $0.05^{\mathrm{b}}$ & $0.02-0.4$ \\
\hline PFNA & 84 & $0.06^{\mathrm{a}}$ & $0.01-0.23$ & 0.06 & $0.02-0.18$ & 0.07 & $0.02-0.23$ & $0.01^{\mathrm{a}}$ & $0.01-0.4$ & 0.09 & $0.02-0.37$ & 0.12 & $0.02-0.5$ \\
\hline PFDA & 20 & 0.01 & $0.01-0.07$ & 0.02 & $0.02-0.04$ & 0.02 & $0.02-0.07$ & 0.01 & $0.01-0.06$ & $0.02^{b}$ & $0.02-0.04$ & $0.02^{b}$ & $0.02-0.0$ \\
\hline NMeFOSAA & 44 & $0.01^{\mathrm{a}}$ & $0.01-0.01$ & 0.01 & $0.01-0.01$ & 0.01 & $0.01-0.08$ & $0.2^{\mathrm{a}}$ & $0.01-1.3$ & 0.2 & $0.01-1.5$ & 0.2 & $0.01-1.3$ \\
\hline NEtFOSAA & 83 & $0.5^{\mathrm{a}}$ & $0.01-1.38$ & 0.5 & $0.01-1.6$ & 0.5 & $0.07-1.3$ & $0.3^{\mathrm{a}}$ & $0.01-1.2$ & $0.3^{\mathrm{b}}$ & $0.01-1.2$ & $0.2^{\mathrm{b}}$ & $0.01-1.2$ \\
\hline FOSA & 13 & $0.01^{\mathrm{a}}$ & $0.01-0.3$ & 0.01 & $0.01-0.3$ & 0.01 & $0.01-0.3$ & $0.01^{\mathrm{a}}$ & $0.01-0.2$ & $0.01^{b}$ & $0.01-0.2$ & $0.01^{\mathrm{b}}$ & $0.01-0.1$ \\
\hline
\end{tabular}

${ }^{\mathrm{a}}$ Statistically significant differences between the countries with the Mann-Whitney $U$ test. ${ }^{\mathrm{b}}$ Statistically significant differences between the cases and controls with the Mann-Whitney $U$ test. 
Table 2 Distribution of different variables (mean or percent) according to PFOA and PFOS concentrations in tertiles. Differences were tested using the $\chi^{2}$ or ANOVA tests.

\begin{tabular}{|c|c|c|c|c|c|c|c|c|}
\hline \multirow[b]{2}{*}{ Variable } & \multirow[b]{2}{*}{$n$} & \multirow[b]{2}{*}{$\begin{array}{c}\text { Percentage } \\
(\%)\end{array}$} & \multicolumn{3}{|c|}{ PFOA (ng/ml) } & \multicolumn{3}{|c|}{ PFOS (ng/ml) } \\
\hline & & & $\begin{array}{c}1 \mathrm{st} \\
(0.65-1.80) \\
n=70\end{array}$ & $\begin{array}{c}\text { 2nd } \\
(1.81-2.63) \\
n=73\end{array}$ & $\begin{array}{c}3 \mathrm{rd} \\
(2.65-5.32) \\
n=72\end{array}$ & $\begin{array}{c}1 \mathrm{st} \\
(1.99-4.84) \\
n=71\end{array}$ & $\begin{array}{c}2 \mathrm{nd} \\
(4.84-6.82) \\
n=72\end{array}$ & $\begin{array}{c}\text { 3rd } \\
(6.90-23.17) \\
n=72 \\
\end{array}$ \\
\hline \multicolumn{9}{|l|}{ Birth weight (g) } \\
\hline$<2500$ & 9 & 4.2 & 5.8 & 2.8 & 4.2 & 4.4 & 4.2 & 4.2 \\
\hline $2500-4500$ & 196 & 92.0 & 89.9 & 93.1 & 93.1 & 92.8 & 90.3 & 93.1 \\
\hline$>4500$ & 8 & 3.8 & 4.4 & 4.2 & 2.8 & 2.9 & 5.6 & 2.8 \\
\hline \multicolumn{9}{|l|}{ Parity } \\
\hline 1st child & 124 & 58.5 & 32.8 & 58.9 & $81.9^{*}$ & 39.7 & 58.3 & $76.4^{*}$ \\
\hline \multicolumn{9}{|c|}{ Gestational age (weeks) } \\
\hline$<37$ & 13 & 6.3 & 9.0 & 5.7 & 4.3 & 7.5 & 5.6 & 5.9 \\
\hline $37-42$ & 189 & 91.3 & 91.0 & 92.9 & 90.0 & 92.5 & 91.7 & 89.7 \\
\hline$>42$ & 5 & 2.4 & 0.0 & 1.4 & 5.7 & 0.0 & 2.8 & 4.4 \\
\hline \multicolumn{9}{|l|}{ Country } \\
\hline Denmark & 59 & 27.4 & 24.3 & 20.6 & 37.5 & 4.2 & 20.8 & $56.9^{*}$ \\
\hline Finland & 156 & 72.6 & 75.7 & 79.5 & 62.5 & 95.8 & 79.2 & 43.1 \\
\hline \multicolumn{9}{|l|}{ Maternal BMI } \\
\hline Mean & 123 & 57.0 & 22.3 & 23.6 & 22.9 & 23.5 & 22.9 & 22.9 \\
\hline Missing & 92 & 43.0 & & & & & & \\
\hline \multicolumn{9}{|l|}{ Maternal age } \\
\hline Mean & 214 & 100 & 30.0 & 30.2 & 29.5 & 29.5 & 30.1 & 30.0 \\
\hline Smoking status & 43 & 20.0 & 18.8 & 21.9 & 19.4 & 21.4 & 20.8 & 18.6 \\
\hline \multicolumn{9}{|l|}{ Birth year } \\
\hline 1997-1999 & 134 & 62.6 & 56.5 & 69.9 & 61.1 & 60.0 & 75.0 & $52.8^{*}$ \\
\hline 2000-2002 & 80 & 37.4 & 43.5 & 30.1 & 38.9 & 40.0 & 25.0 & 47.2 \\
\hline
\end{tabular}

$* P<0.05$.

semen quality have been described as different manifestations of a syndrome with a common underlying embryonal etiology 'the testicular dysgenesis syndrome (TDS)' (Skakkebaek et al. 2001). In accordance with the TDS hypothesis, the incidences of testicular cancer, poor semen quality, and hypospadias have also been reported to be higher in Denmark than in Finland (Skakkebaek et al. 2006).

We did not find any statistical associations between prenatal PFOA and PFOS exposure and congenital cryptorchidism (using cord blood levels as biomarker); however, this may be due to lack of power due to limited sample size. However, it was not possible to include more cases, as data were collected prospectively and cord blood samples were collected before information on cryptorchidism at the age of 3 months was obtained. Controls were matched by date of birth, gestational age, parity, maternal diabetes, and smoking status and also had to have cord blood samples stored, which made it impossible to identify more controls. Our findings suggest that differences in PFAS exposure do not contribute to the difference in prevalence of TDS-related conditions between Denmark and Finland, even though Danish women seem to be more exposed. On the contrary, when only the Finnish samples were analyzed, the data suggested a negative association between PFOA exposure and congenital cryptorchidism risk.

The advantages of the nested case-control design compared with those of a classic case-control study are that data are collected prospectively and questionnaire information is obtained before the outcome. Moreover, the PFAS exposure levels of the participants were unknown both to the pregnant women and to the examining doctors. The participation rate of the cohort study was $22-24 \%$. Women from higher social classes were over-represented in the study. In Finland, the incidence of cryptorchidism in the prenatally recruited cohort was the same as that for all children born in the study hospital, suggesting that the recruitment did not cause an over-representation of cases with cryptorchidism. American women from higher social classes have been reported to have higher levels of PFAS than those from lower social classes (Nelson et al. 2006); however, no differences in PFOA and PFOS exposure levels according to social class were found in Denmark (Fei et al. 2007). We adjusted for other potential confounders including strong predictors for cryptorchidism (birth weight and gestational age); however, we cannot exclude residual confounding by other unknown or unreported confounders.

PFOA and PFOS levels in cord blood have been found to represent $30-79 \%$ of the levels in maternal serum, but with a good correlation. Short-chained PFASs are found in higher proportions in cord blood than long-chained ones (Ehresman et al. 2007, Gutzkow et al. 2012). A few studies have measured PFOA and PFOS levels in serum samples collected from Danish pregnant and pre-pregnant women (Fei et al. 2009, Vestergaard et al. 2012), but few studies have measured PFAS levels in cord blood. Fei et al. (2007) measured PFOA and PFOS levels in 
Table 3 Crude and adjusted ${ }^{\mathrm{a}}$ odds ratios (ORs) and 95\% Cls for cryptorchidism according to PFOA and PFOS levels among boys in Denmark and Finland. PFOA and PFOS levels were analyzed as continuous variables or in tertiles.

\begin{tabular}{|c|c|c|c|c|c|c|}
\hline \multirow{2}{*}{$\begin{array}{l}\text { PFOA and PFOS } \\
(\mathrm{ng} / \mathrm{ml})\end{array}$} & \multicolumn{3}{|c|}{ Crude OR } & \multicolumn{3}{|c|}{ Adjusted OR $^{\mathrm{a}}$} \\
\hline & All $(n=215)$ & Denmark $(n=59)$ & Finland $(n=156)$ & All $(n=205)$ & Denmark $(n=55)$ & Finland $(n=148)$ \\
\hline $\mathrm{PFOA}^{\mathrm{b}}$ & $0.50(0.25-1.02)$ & $0.65(0.15-2.69)$ & $0.45(0.20-1.04)$ & $0.51(0.21-1.20)$ & $1.14(0.19-6.95)$ & $0.35(0.12-1.02)$ \\
\hline PFOS $^{b}$ & $0.69(0.40-1.20)$ & $0.96(0.25-3.71)$ & $0.54(0.26-1.14)$ & $0.83(0.44-1.58)$ & $1.30(0.27-6.39)$ & $0.55(0.23-1.32)$ \\
\hline PFOA 1st & Reference & Reference & Reference & Reference & Reference & Reference \\
\hline PFOA 2nd & $0.55(0.28-1.07)$ & $1.05(0.26-4.32)$ & $0.46(0.21-0.98)$ & $0.58(0.28-1.22)$ & $1.79(0.35-9.30)$ & $0.42(0.17-1.02)$ \\
\hline PFOA 3rd & $0.45(0.23-0.88)$ & $0.41(0.12-1.42)$ & $0.48(0.22-1.09)$ & $0.46(0.20-1.02)$ & $0.78(0.17-3.61)$ & $0.35(0.12-0.99)$ \\
\hline$P$ trend & 0.02 & 0.13 & 0.07 & 0.06 & 0.83 & 0.04 \\
\hline PFOS 1st & Reference & Reference & Reference & Reference & Reference & Reference \\
\hline PFOS 2nd & $0.52(0.27-1.02)$ & $3.00(0.22-40.93)$ & $0.41(0.20-0.84)$ & $0.70(0.34-1.46)$ & $3.55(0.20-62.49)$ & $0.52(0.23-1.16)$ \\
\hline PFOS 3rd & $0.73(0.38-1.42)$ & $1.73(0.14-20.58)$ & $0.85(0.36-2.00)$ & $0.83(0.39-1.78)$ & $2.34(0.16-34.67)$ & $0.75(0.26-2.11)$ \\
\hline$P$ trend & 0.36 & 0.76 & 0.34 & 0.64 & 0.97 & 0.38 \\
\hline
\end{tabular}

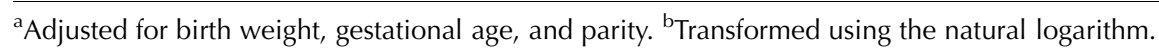

50 cord blood samples within The Danish National Birth Cohort (DNBC) from 1996 to 2002 and found levels comparable to the cord blood levels in the Danish samples observed in our study. A Norwegian study carried out using cord blood samples collected from 1997 to 1998 found lower median PFOA and PFOS levels than those found in our study, whereas the more recently introduced PFASs PFHxS, PFDA, and PFNA were found in higher concentrations (Gutzkow et al. 2012).

Male reproductive organ programming window occurs at gestational weeks 8-15 (Welsh et al. 2008), and we measured PFAS levels in cord blood months later. Monroy et al. (2008) measured PFAS levels in blood in the second and third trimesters and in cord blood at delivery and found lower levels in cord blood than in maternal blood. We may, therefore, have underestimated PFAS exposure during the vulnerable development period; however, this underestimation is likely to be similar among cases and controls, thereby underestimating a possible association. In addition, the second phase of testicular descent into the scrotum occurs late in pregnancy and is also androgen dependent whereby late pregnancy exposure may be relevant (Bay et al. 2011) and cord blood may help better estimate what the fetus is exposed to than maternal blood.

PFASs have been shown to exhibit endocrine-disrupting abilities, but mechanisms are not well known (Jensen \& Leffers 2008). Animal studies have suggested that PFASs adversely affect reproduction. PFOS exposure in female rats affects estrous cyclicity (Austin et al. 2003). In utero PFOS exposure in rats and mice has been shown to cause reduced litter size, reduced body weight, and delayed development, but there are no reports of increase in malformations (Lau et al. 2007). In utero PFOA exposure has been shown to be associated with altered mammary gland development in mice (White et al. 2007). PFOA exposure in adult rats has been found to cause lower testosterone levels and increasing estradiol levels. Both PFOA and PFOS affect Leydig cell function through different modes of action in rats and humans (Ye et al. 2011). Other PFASs have estrogenic effects in cell cultures (Jensen \& Leffers 2008). However, the results from human studies have been inconsistent (Olsen et al. 2009). The DNBC included serum samples collected from 1400 pregnant women and found an association between PFOA, but not PFOS, exposure and low birth weight (Fei et al. 2007). A recent American study in a population exposed to PFOA-contaminated drinking water has found no association with pregnancy outcome (preterm birth, gestational age, birth weight, and stillbirth; Savitz et al. 2012), similar to the findings reported by Monroy et al. (2008). However, Savitz et al. estimated pregnancy exposure levels by extrapolation from later samples and Monroy et al. (2008) included only 101 women. Stein et al. (2009) found that PFOA and PFOS exposure levels were associated with a higher risk of pre-eclampsia in pregnancy and birth defects (not specified) in a community with high PFOA exposure.

PFOA and PFOS have been phased out and replaced by the more recent PFASs (e.g. PFNA and PFDA) due to the growing knowledge about their adverse health effects. In a Swedish study, PFHxS exposure level in women was found to increase $8.3 \%$ per year from 1996 to 2010, whereas PFDA and PFNA levels in the same period increased 3.8 and $4.3 \%$ per year (Glynn et al. 2010). We found that PFOA and PFOS levels decreased with increasing year of birth. More Finnish cases than controls were recruited in later birth years, which could explain why Finnish cases were lower exposed than controls. The analyses were, therefore, repeated among Finnish cases and controls recruited from 1997 to 1999 and differences in exposure levels between cases and controls were attenuated.

In conclusion, we found measurable levels of PFOA and PFOS in all the cord blood samples and higher levels of all PFASs in the Danish cord blood samples than in the Finnish cord blood samples. Cord blood PFOA, PFOS, and PFNA levels were not significantly associated with congenital cryptorchidism; however, we cannot exclude 
that the lack of statistical associations may be due to the limited sample size. Future studies should, therefore, include larger samples and also measure the levels of more recent PFASs.

\section{Declaration of interest}

D Vesterholm Jensen, J Christensen, H E Virtanen, J Toppari, C W Veje, and P Grandjean have no relationship with any sponsors of the COW meeting, Danish Ministry of Environment and Environment Protection Agency. N E Skakkebæk, A-M Andersson, $F$ Nielsen, and $T \mathrm{~K}$ Jensen have received non-profit grants from the Danish Ministry of Environment EPA for this study. In addition, A-M Andersson is the leader of the Danish Center for Endocrine Disruptors under the Danish Ministry of Environment and Environment Protection Agency. K M Main has received non-profit grants from the Danish Ministry of Environment - EPA for two other scientific studies not related to the study mentioned in this article. T K Jensen has also been a member of an advisory group related to human studies on pesticide effects.

\section{Funding}

This work was supported by the Danish Agency for Science, Technology and Innovation (09-067180), Academy of Finland, the Turku University Hospital, Sigrid Juselius Foundation, the European Commission (QLK4-CT-1999-01422, QLK4-CT2001-00269, QLK4-2002-00603, FP7/2008-2012: DEER 212844), the Danish Council for Strategic Research, Program Commission on Health, Food and Welfare (project 2101-080058), Kirsten and Freddy Johansens Fund (grant number 95-103-72087), Rigshospitalet (grant number 961506336), the Danish Ministry of Health, and the Danish Environmental Protection Agency. This article is based on work presented at the 7th Copenhagen Workshop on Endocrine Disrupters, which was supported by the Danish Ministry of the Environment Environmental Protection Agency. The publication of this special issue was supported by the Society for Reproduction and Fertility.

\section{References}

Austin ME, Kasturi BS, Barber M, Kannan K, MohanKumar PS \& MohanKumar SM 2003 Neuroendocrine effects of perfluorooctane sulfonate in rats. Environmental Health Perspectives 111 1485-1489. (doi:10.1289/ehp.6128)

Bay K, Main KM, Toppari J \& Skakkebaek NE 2011 Testicular descent: Insl3, testosterone, genes and the intrauterine milieu. Nature Reviews. Urology 8 187-196. (doi:10.1038/nrurol.2011.23)

Boisen KA, Kaleva M, Main KM, Virtanen HE, Haavisto AM, Schmidt IM, Chellakooty M, Damgaard IN, Mau C, Reunanen M et al. 2004 Difference in prevalence of congenital cryptorchidism in infants between two Nordic countries. Lancet 363 1264-1269. (doi:10.1016/S01406736(04)15998-9)

Ehresman DJ, Froehlich JW, Olsen GW, Chang SC \& Butenhoff JL 2007 Comparison of human whole blood, plasma, and serum matrices for the determination of perfluorooctanesulfonate (PFOS), perfluorooctanoate (PFOA), and other fluorochemicals. Environmental Research 103 176-184. (doi:10.1016/j.envres.2006.06.008)
Fei C, McLaughlin JK, Tarone RE \& Olsen J 2007 Perfluorinated chemicals and fetal growth: a study within the Danish National Birth Cohort. Environmental Health Perspectives 115 1677-1682. (doi:10.1289/ ehp.10506)

Fei C, McLaughlin JK, Lipworth L \& Olsen J 2009 Maternal levels of perfluorinated chemicals and subfecundity. Human Reproduction 24 1200-1205. (doi:10.1093/humrep/den490)

Glynn A, Berger U, Bignert A, Ullah S, Aune M, Lignell S \& Darnerud PO 2010 Perfluorinated alkyl acids in blood serum from primiparous women in Sweden: serial sampling during pregnancy and nursing, and temporal trends 1996-2010. Environmental Science \& Technology 46 9071-9079. (doi:10.1021/es301168c)

Gutzkow KB, Haug LS, Thomsen C, Sabaredzovic A, Becher G \& Brunborg G 2012 Placental transfer of perfluorinated compounds is selective - a Norwegian mother and child sub-cohort study. International Journal of Hygiene and Environmental Health 215 216-219. (doi:10. 1016/j.ijheh.2011.08.011)

Haug LS, Thomsen C \& Becher G 2009 A sensitive method for determination of a broad range of perfluorinated compounds in serum suitable for large-scale human biomonitoring. Journal of Chromatography. A 1216 385-393. (doi:10.1016/j.chroma.2008.10.113)

Jensen AA \& Leffers H 2008 Emerging endocrine disrupters: perfluoroalkylated substances. International Journal of Andrology 31 161-169. (doi:10.1111/j.1365-2605.2008.00870.x)

Joensen UN, Veyrand B, Antignac JP, Blomberg Jensen M, Petersen JH, Marchand P, Skakkebæk NE, Andersson AM, Le Bizec B \& Jørgensen N 2013 PFOS (perfluorooctanesulfonate) in serum is negatively associated with testosterone levels, but not with semen quality, in healthy men. Human Reproduction 28 599-608. (doi:10.1093/humrep/ des425)

Krysiak-Baltyn K, Toppari J, Skakkebaek NE, Jensen TS, Virtanen HE, Schramm KW, Shen H, Vartiainen T, Kiviranta H, Taboureau $O$ et al. 2010 Country-specific chemical signatures of persistent environmental compounds in breast milk. International Journal of Andrology 33 270-278. (doi:10.1111/j.1365-2605.2009.00996.x)

Lau C, Anitole K, Hodes C, Lai D, Pfahles-Hutchens A \& Seed J 2007 Perfluoroalkyl acids: a review of monitoring and toxicological findings. Toxicological Sciences 99 366-394. (doi:10.1093/toxsci/ kfm128)

Lindstrom AB, Strynar MJ \& Libelo EL 2011 Polyfluorinated compounds: past, present, and future. Environmental Science \& Technology 45 7954-7961. (doi:10.1021/es2011622)

Monroy R, Morrison K, Teo K, Atkinson S, Kubwabo C, Stewart B \& Foster WG 2008 Serum levels of perfluoroalkyl compounds in human maternal and umbilical cord blood samples. Environmental Research 108 56-62. (doi:10.1016/j.envres.2008.06.001)

Nelson JW, Scammell MK, Hatch EE \& Webster TF 2006 Social disparities in exposures to bisphenol A and polyfluoroalkyl chemicals: a crosssectional study within NHANES 2003-2006. Environmental Health 11 10. (doi:10.1186/1476-069X-11-10)

Olsen GW, Butenhoff JL \& Zobel LR 2009 Perfluoroalkyl chemicals and human fetal development: an epidemiologic review with clinical and toxicological perspectives. Reproductive Toxicology 27 212-230. (doi:10.1016/j.reprotox.2009.02.001)

Rantakokko P, Main KM, Wohlfart-Veje C, Kiviranta H, Airaksinen R, Vartiainen T, Skakkebæk NE, Toppari J \& Virtanen HE 2013 Association of placenta organotin concentrations with congenital cryptorchidism and reproductive hormone levels in 280 newborn boys from Denmark and Finland. Human Reproduction 28 1647-1660. (doi:10.1093/humrep/ det040)

Savitz DA, Stein CR, Bartell SM, Elston B, Gong J, Shin HM \& Wellenius GA 2012 Perfluorooctanoic acid exposure and pregnancy outcome in a highly exposed community. Epidemiology 23 386-392. (doi:10.1097/ EDE.0b013e31824cb93b)

Scorer CG 1964 The descent of the testis. Archives of Disease in Childhood 39 605-609. (doi:10.1136/adc.39.208.605)

Shen H, Main KM, Andersson AM, Damgaard IN, Virtanen HE, Skakkebaek NE, Toppari J \& Schramm KW 2008 Concentrations of persistent organochlorine compounds in human milk and placenta are higher in Denmark than in Finland. Human Reproduction 23 201-210. 
Skakkebaek NE, Rajpert-De Meyts E \& Main KM 2001 Testicular dysgenesis syndrome: an increasingly common developmental disorder with environmental aspects. Human Reproduction 16 972-978. (doi:10. 1093/humrep/16.5.972)

Skakkebaek NE, Jørgensen N, Main KM, Rajpert-De Meyts E, Leffers H, Andersson AM, Juul A, Carlsen E, Mortensen GK, Jensen TK et al. 2006 Is human fecundity declining? International Journal of Andrology 29 2-11. (doi:10.1111/j.1365-2605.2005.00573.x)

Stein CR, Savitz DA \& Dougan M 2009 Serum levels of perfluorooctanoic acid and perfluorooctane sulfonate and pregnancy outcome. American Journal of Epidemiology 170 837-846. (doi:10.1093/aje/kwp212)

Vestergaard S, Nielsen F, Andersson AM, Hjøllund NH, Grandjean P, Andersen HR \& Jensen TK 2012 Association between perfluorinated compounds and time to pregnancy in a prospective cohort of Danish couples attempting to conceive. Human Reproduction 27 873-880. (doi:10.1093/humrep/der450)

Washino N, Saijo Y, Sasaki S, Kato S, Ban S, Konishi K, Ito R, Nakata A Iwasaki Y, Saito K et al. 2009 Correlations between prenatal exposure to perfluorinated chemicals and reduced fetal growth. Environmental Health Perspectives 117 660-667. (doi:10.1289/ehp.11681)
Welsh M, Saunders PT, Fisken M, Scott HM, Hutchison GR, Smith LB \& Sharpe RM 2008 Identification in rats of a programming window for reproductive tract masculinization, disruption of which leads to hypospadias and cryptorchidism. Journal of Clinical Investigation 118 1479-1490. (doi:10.1172/JCI34241)

White SS, Calafat AM, Kuklenyik Z, Villanueva L, Zehr RD, Helfant L, Strynar MJ, Lindstrom AB, Thibodeaux JR, Wood C et al. 2007 Gestational PFOA exposure of mice is associated with altered mammary gland development in dams and female offspring. Toxicological Sciences 96 133-144. (doi:10.1093/toxsci/kfl177)

Ye L, Su ZJ \& Ge RS 2011 Inhibitors of testosterone biosynthetic and metabolic activation enzymes. Molecules 16 9983-10001. (doi:10. 3390/molecules16129983)

Received 12 September 2013

First decision 14 October 2013

Revised manuscript received 15 October 2013

Accepted 11 November 2013 